\title{
Evaluation of the Effects of Chitosan on Immune Responses due to Infectious Bursal Disease Virus (IBDV) Vaccine in Chicken
}

\author{
Armandei Sadi $^{1,2} \oplus$, Shahsavandi Shahla*1 ${ }^{1}$, Ebrahimi Mohammad Majid ${ }^{1} \oplus$ \\ ${ }^{1}$ Razi Vaccine and Serum Research Institute, Agricultural Research Education and Extension Organization, Karaj, Iran. ${ }^{2}$ Islamic \\ Azad University, Karaj Branch, Iran
}

\begin{tabular}{l}
\hline A R T I C L E I N F O \\
\hline Original Article \\
VacRes, 2019 \\
Vol. 6, No.2, 18-22 \\
Received: March 02, 2020 \\
Accepted:May 06, 2020 \\
Pasteur Institute of Iran \\
*Corresponding Author: \\
Shahsavandi Shahla. Razi Vaccine \\
and Serum Research Institute, \\
Agricultural Research Education and \\
Extension Organization, Karaj, Iran \\
Email: s.shahsavandi @ rvsri.ac.ir \\
Tel/Fax: 02634570038-46 / \\
02634552194
\end{tabular}

KEYWORDS: Infectious Bursal Disease Virus (IBDV), inactivated antigen, chitosan, adjuvant

\author{
A B S T R A C T
}

Introduction: Inactivated Infectious Bursal Disease Virus (IBDV) is administrated against Infectious Bursal Disease (IBD). Chitosan biopolymer is capable of inducing proper immune responses to an antigen while being non-toxic and degradable. In this study, the effect of chitosan on improving the humoral immune responses against IBD virus (IBDV) was investigated. Methods: The antigen was prepared by inoculating IBDV in embryonated chicken eggs and inactivation by ethylenimine. Chitosan solutions at final concentrations of $0.5 \%$ and $1 \%$ were made and used as an adjuvant. One-day-old chickens were randomly divided in 8 groups and received intramuscularly different profiles of the inactivated antigen and chitosan solution. Serum samples were collected before the prime and booster injection as well as at the defined intervals and assayed by ELISA and serum neutralization tests. Results: After 2-dose intramuscular immunization with inactivated IBDV in combination with chitosan, significant increases $(\mathrm{P}<0.05)$ in antibody titers were observed compared with other administration groups. The addition of $1 \%$ chitosan to inactivated IBDV resulted in higher level of neutralizing antibody titer than $0.5 \%$ chitosan. However, the difference in the enhancing of antibody titers and the neutralization index between these concentrations was not significant. Conclusion: These data revealed that chitosan as an antigen carrier has the potential to enhance specific immune responses induced by inactived IBDV.

\section{Citation:}

ebrahimi M M, Armandei S, Shahsavandi S. Evaluation of the Effects of Chitosan on Immune Responses due to Infectious Bursal Disease Virus (IBDV) Vaccine in Chicken. vacres. 2019; 6 (2) :18-22. DOI: $10.29252 /$ vacres.6.2.18

\section{INTRODUCTION}

Infectious Bursal Disease (IBD) or Gumboro is a highly contagious and immunosuppressive disease of young chickens. The causative agent, Infectious Bursal Disease Virus (IBDV) belongs to Avibirnavirus genus of Birnaviridae family. The bisegmented genome of the virus is divided into two segments, namely $\mathrm{A}$ and $\mathrm{B}$. The open reading frame of segment $\mathrm{A}$ encodes for a polyprotein (pVP2-VP4-VP3) and for a nonstructural protein VP5. The segment B encodes an RNAdependent RNA polymerase (VP1) which plays a key role in encapsidation of the viral particles [1-3]. IBDV replicates in bursa of Fabricius as the target organ. Spread of the virus in kidneys and muscles leads to pathognomonic IBD clinical signs $[4,5]$.

Since its emergence about 60 years ago, IBD still poses an economic threat to the poultry industry. Prevention of the infection at an early chicken age is essential to control the immunosuppressive effect of IBDV. Live-attenuated IBDV vaccines are used in breeder flocks as a primary vaccine and inactivated vaccines are administered for boosting the specific immune responses and extension of the maternal immunity [6, 4]. Because the inactivated vaccines produce a weaker immune response, adjuvants should be required to provide effective immunity against the inactivated virus. Generally, vaccine adjuvants are chemical substances that enhance antigen delivery to the immune cells, stimulate the immune responses to produce more antibodies and longer-lasting immunity. Water in oil emulsions are used as adjuvants in order to enhance the immunogenicity of inactivated vaccines when long term immunity is required. Post-immunization reactions such as edema and necrosis at the injection sites may limit the application of these adjuvants. Hence, developing a simple, tolerable, safe, and cost-efficient adjuvant formulation that induces both Th1 and Th2 types of the immune responses will be of great value in the poultry industry $[7,8]$.

A number of polysaccharides and their derivatives possess adjuvant properties and promote the antigen-specific immune system by acting as pathogen-associated molecular patterns. Such patterns can be recognized by receptors 
including Toll-like receptors and Nod-like receptors on the immune cells which can then trigger the innate immunity and regulate the adaptive immunity [9]. Chitosan, obtained by partial deacetylation of chitin is the second most common natural polysaccharide. Having properties such as being nontoxic, biocompatible and biodegradable make chitosan a proper adjuvant for injectable and mucosal vaccines [10]. Numerous studies in mammalian and avian species have demonstrated that chitosan is an effective and safe adsorption enhancer to improve both the humoral and the cell-mediated immune responses [1113]. Based on its principal mechanisms of action, this biopolymer can control the release and storage of the antigens while increasing the vaccine delivery by making the antigen a target for the antigen-presenting cells. In this study, the adjuvant impact of chitosan on immunogenicity of inactivated IBDV antigen was investigated using specificpathogen-free (SPF) white Leghorn chicken.

\section{MATERIALS and METHODS}

\section{Inactivated IBDV Antigen Preparation and Validation}

The IBDV antigen was prepared by injection of IBD07IR intermediate strain [14] into chorio-allantoic membrane of 911-day-old SPF chicken embryos (obtained from Razi Institute, Karaj, Iran). After 5 days incubation at $37^{\circ} \mathrm{C}$, the amnioallantoic fluid and infective embryo were harvested, grinded, and clarified by centrifugation at $5000 \mathrm{rpm}$ for $30 \mathrm{~min}$ at $4{ }^{\circ} \mathrm{C}$. The virus titer was calculated by Spearman-Karber method based on the following formula:

$\log 1050 \%$ end point dilution $=-\left(\mathrm{x} 0-\mathrm{d} / 2+\mathrm{d} \sum \mathrm{ri} / \mathrm{ni}\right)$ in which $\mathrm{x} 0=\log 10$ of the lowest concentration at which all eggs are positive; $\mathrm{d}=\log 10$ of the dilution factor; $\mathrm{ni}=$ number of eggs used in each individual dilution; $r i=$ number of positive eggs (out of ni) [15] and expressed as the embryo infective dose $50\left(\mathrm{EID}_{50}\right)$.

The viral fluid was inactivated with $3 \mathrm{mM}$ of binary ethylene imine (Sigma-Aldrich, Germany) at $37^{\circ} \mathrm{C}$ for $16 \mathrm{~h}$. The inactivated IBDV was inoculated sequentially 3 times into five 9-11-day-old SPF chicken embryos to ensure the inactivation. Parts of the inactivated IBDV antigen were cultivated on soya-bean casein digest, fluid thioglycollate, sabouraud dextrose agar, and PPLO (all purchased from Difco, UK) and incubated at specified temperatures for at least 14 days to detect bacteria, fungi, and mycoplasma contaminations. Safety of the inactivated IBDV was detected by injection of two doses of the antigen into 10 chickens. Birds were daily monitored for general reactions and clinical signs of IBD for 21 days [6].

\section{Chitosan Solution Formulation}

The low Mw chitosan consisting of 2 units N-acetyl-Dglucosamine and D-glucosamine was purchased from SigmaAldrich, Germany. Chitosan solution at concentration of $1.0 \%$ was prepared by mixing $1 \mathrm{~g}$ of chitosan powder in $90 \mathrm{ml}$ distilled water and $10 \mathrm{ml}$ acetic acid $0.1 \mathrm{M}$ (Merck, Germany) with a high-speed stirrer. This procedure was continued for 30 min or until dissolution completed [16]. A $0.5 \%$ chitosan solution was also prepared.

\section{Ethics Statement}

All animal experiments were carried out according to the standard approved animal care guidelines and protocols, approved by the animal ethics committee of Islamic Azad University, Karaj Branch, N IR.IU.K.REC. 1397.8).

\section{Chicken Immunization}

Sixty 1-day-old SPF chickens were randomly divided into 8 groups and tagged for vaccination trial as described in Table 1. Feed and water were supplied ad-libitum during the experiment. Chickens in all groups received $0.5 \mathrm{ml}$ of the related samples subcutaneously at the back of the neck. For groups $\mathrm{D}$ and $\mathrm{E}$, equal quantities (w/w) of each $0.5 \%$ and $1 \%$ chitosan solutions were mixed with the inactivated IBDV antigen at the titer of 7.3 EID $_{50}$ before the injection. Chickens in these groups were received booster regimens, 1 week after the prime vaccination.

Table 1. Treatment groups in IBDV and chitosan vaccination experiment.

\begin{tabular}{|c|c|c|c|}
\hline \multicolumn{2}{|c|}{ Groups } & \multirow{2}{*}{$\begin{array}{c}\begin{array}{c}\text { chickens/ } \\
\text { group }\end{array} \\
5\end{array}$} & \multirow{2}{*}{$\begin{array}{c}\text { Treatment } \\
\text { phosphate-buffered saline (PBS) }\end{array}$} \\
\hline \multirow{4}{*}{ 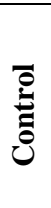 } & $\mathrm{C1}$ & & \\
\hline & $\mathrm{C2}$ & 5 & $0.5 \%$ chitosan solution \\
\hline & $\mathrm{C3}$ & 5 & $1 \%$ chitosan solution \\
\hline & C4 & 5 & inactivated IBDV antigen \\
\hline \multirow{4}{*}{ : } & $\mathbf{A}$ & 10 & $\begin{array}{l}0.5 \% \text { chitosan in prime and } \\
\text { inactivated IBDV antigen in boost }\end{array}$ \\
\hline & B & 10 & $\begin{array}{l}1 \% \text { chitosan in prime and } \\
\text { inactivated IBDV antigen in boost }\end{array}$ \\
\hline & D & 10 & $\begin{array}{l}\text { combination of } 0.5 \% \text { chitosan and } \\
\text { inactivated IBDV antigen twice } \\
\text { with one-week-interval }\end{array}$ \\
\hline & $\mathbf{E}$ & 10 & $\begin{array}{l}\text { combination of } 1 \% \text { chitosan and } \\
\text { inactivated IBDV antigen twice } \\
\text { with one-week-interval }\end{array}$ \\
\hline
\end{tabular}

\section{Serological Evaluation}

Blood was taken from wing vein of all the chickens at 1 , 2, 3, and 4 weeks, post-vaccination. Serum was separated from the blood clot by placing the syringe in approximately $2 \mathrm{~h}$ at $37^{\circ} \mathrm{C}$. Then the serum was transferred to a microfuge tube and centrifuged at $1000 \mathrm{x} \mathrm{g}$ for $5 \mathrm{~min}$. To quantify the level of specific IBDV antibodies, ELISA was done on serum collected from each chicken using IDEXX IBD Ab Test commercial kit (IDEXX Laboratories, Inc., The Netherlands), following the manufacturer's direction. In order to evaluate IBDV-specific serum neutralizing (SN) antibody levels, serial 10-fold dilutions $(10-1-10-7)$ of IBDV were prepared, and each dilution was mixed with the same volume of pooled sera [6]. Five embryonated SPF eggs were inoculated with $0.2 \mathrm{ml}$ of the virus-serum mixtures at each serial dilution and the eggs were incubated at $37^{\circ} \mathrm{C}$. Seven days later, pathological changes were observed and the neutralizing index (NI) was calculated as the difference between the $\log$ titer of the negative serum-virus mixture and the log titer of the positive serum-virus mixture.

\section{Statistical Analysis}

Statistical differences between the experimental groups were determined by Student's t-tests which were conducted using SPSS ver. 22. Statistical significance was considered when $P$ value was less than 0.05 . 


\section{RESULTS}

\section{Effects of IBDV on embryonated eggs}

IBDV was replicated in embryonated eggs within 4 to 5 days post-inoculation and exhibited severe hemorrhage and insufficient embryo development (Fig. 1). Titer of the virus in the harvested antigen was calculated as $7.9 \quad \mathrm{EID}_{50} / \mathrm{ml}$. Microbiological examinations revealed the IBDV antigen is free from the presence of bacteria, fungi, and mycoplasma. None of the chickens injected with inactivated IBDV showed the clinical signs of the disease at 3 passages. These confirmed the sterility and safety of the inactivated antigen.

\section{Humoral Immune Response}

The evaluation of humoral immune response indicated that the mean ELISA antibody titer is higher in group $\mathrm{E}$ (immunized with inactivated IBDV with chitosan 1\%) than other treatment groups and the difference between this group and the control groups was significant $(\mathrm{P}<0.05)$. Groups $\mathrm{A}$ and $\mathrm{B}$ that primed with chitosan at 0.5 and $1 \%$ concentrations then boosted with inactivated IBDV antigen had high levels of antibodies compared to the control groups (Fig. 2). Compared to $\mathrm{A}, \mathrm{B}$, and $\mathrm{C} 4$ groups, a significant increase in antibody titer observed in groups $\mathrm{D}$ and $\mathrm{E}$ which were vaccinated twice.

\section{Neutralization index of the immunized chicken}

Similar to the ELISA result, the NI was significantly higher $(\mathrm{P}<0.05)$ in chickens immunized with inactivated IBDV with chitosan at 0.5 and $1 \%$ concentrations, compared to the other chicken groups (Fig. 3).

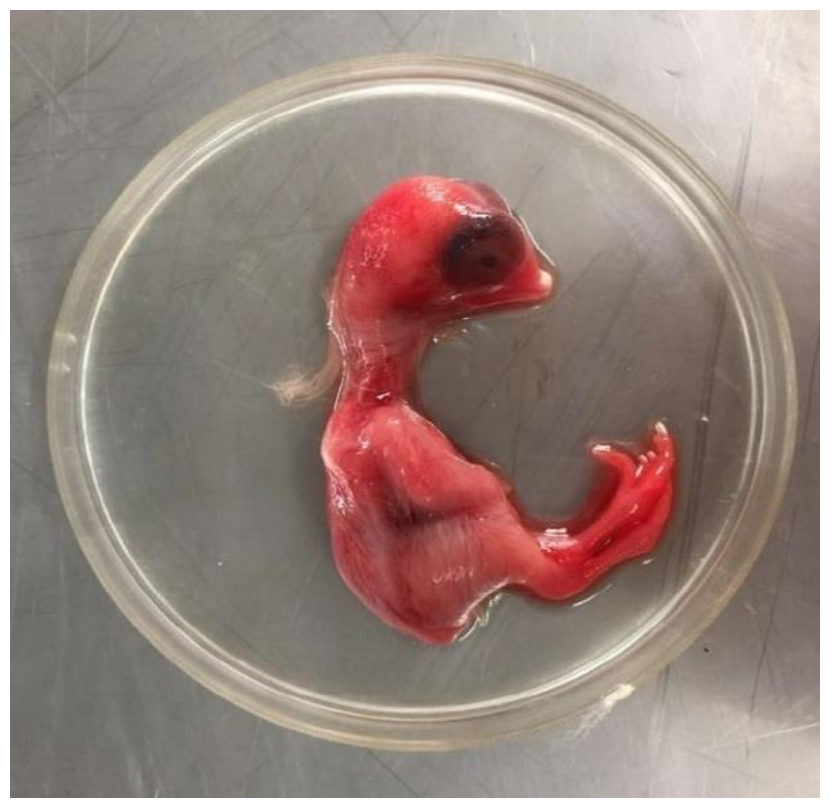

Fig. 1. Replication of IBDV in embryonated eggs caused severe hemorrhage and insufficient embryo development signs.

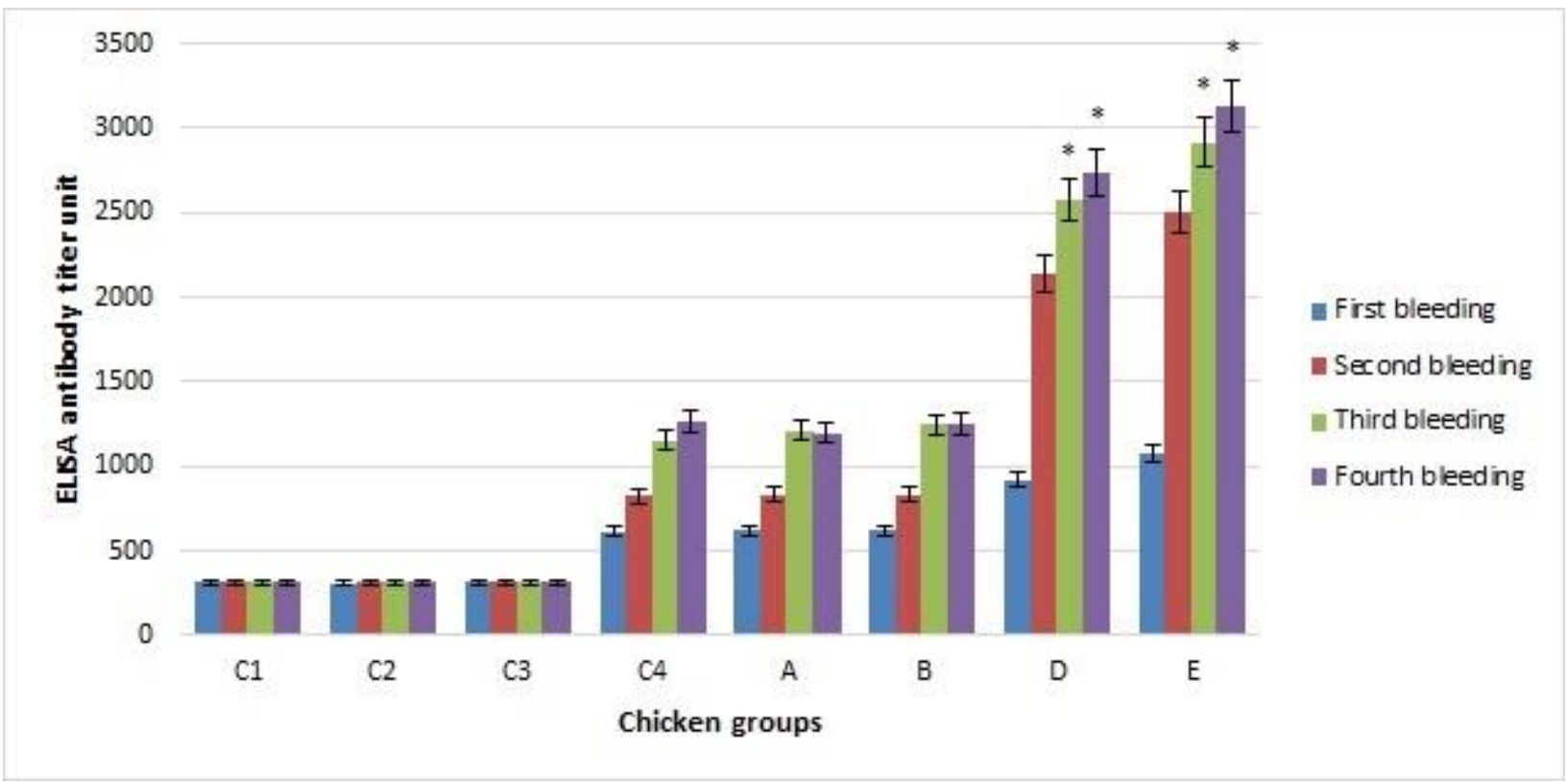

Fig. 2. The ELISA specific infectious bursal disease virus antibody response detection in the groups of chicken administered with different chitosan and inactivated virus antigen profiles. Asterisks $(*)$ denote significant differences $(\mathrm{P}<0.05)$. 


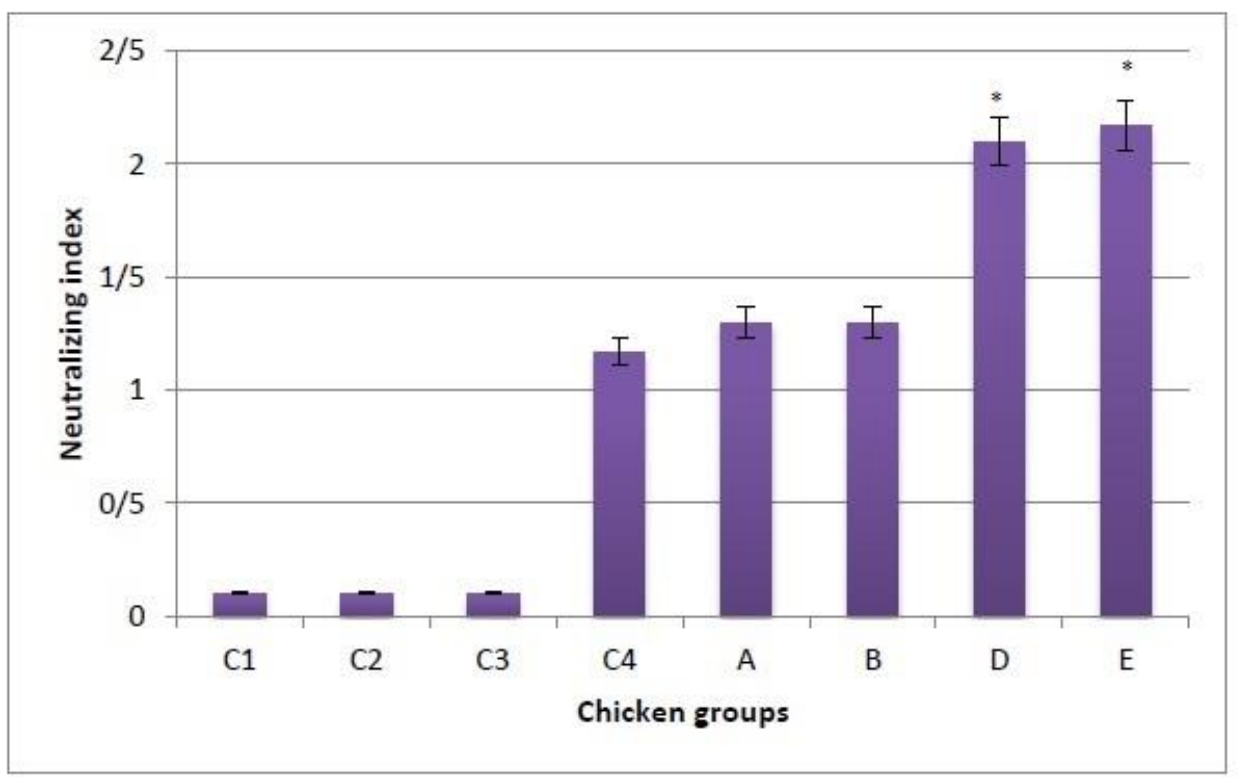

Fig. 3. Neutralizing index detection in the groups of chicken administered with different chitosan and inactivated virus antigen profiles. Asterisks $(*)$ denote significant differences $(\mathrm{P}<0.05)$.

Chickens in groups $\mathrm{C} 4, \mathrm{~A}$, and B showed an NI of similar value with a range of 1.2 to 1.4 . Based on the serological results, the inactivated antigen in combination with $1 \%$ chitosan stimulates IBDV specific immune response and induces high levels of neutralizing antibodies which may confer full protection against the disease.

\section{DISCUSSION}

The occurrence of IBD in intensive poultry production systems have led to the immunosuppression and further dissemination of other viral diseases. The main method of prevention and controlling the immunosuppressive effect of IBDV is vaccination of the chickens at an early age. Administration of inactivated IBDV vaccine can promote the specific immune responses, mediated by neutralizing antibodies which maintain at high levels through 10 months of lay $[17,4]$. The choice of the adjuvant is an important factor for improving the ability of the inactivated vaccine to provide longlasting protection against the infection $[18,8]$. In our study, SPF chickens were intramuscularly administrated with inactivated IBDV antigen and various concentrations of chitosan in different regimens. Our results indicated that only co-administration of $0.5 \%$ and $1 \%$ chitosan with inactivated IBDV induced a specific immune response in the chickens. However, the higher antibody responses produced when $1 \%$ chitosan was used.

Most studies have been focused on the viscosity and mucoadhesive properties of chitosan and candidate it as an effective intranasal adjuvant in mammalian models. In mucosal administration, chitosan retains the antigen in nasal passages and open epithelial-cell tight junctions then the free antigens can enter and bind properly to the dendritic cells (DC) and activate them [13]. The DC activation may alter depending on the size, $\mathrm{Mw}$ and chemical modification for the solubility of chitosan [19]. Through mucosal vaccination, chitosan could promote the production of the Th1-associated cytokine IFN- $\gamma$ whereas the Th2-associated cytokines limitedly produced [17].
Reconstitution of a live Newcastle disease vaccine with chitosan solution have been shown to have a positive effect on cell-mediated immunity by improving the specific IFN- $\gamma$ production without detectable effect on the humoral immunity in poultry [17]. The lesser ability in inducing the humoral immunity has been mentioned with respect to mucosal vaccination than the injected vaccines. In contrast, the formulated inactivated antigens with chitosan have been shown to induce adaptive Th2 immune responses and have produced prolonged and higher levels of antigen-specific antibody titers when injected [20,11]. Moreover, intramuscular administration of mice with $0.5 \%$ chitosan and an inactivated influenza vaccine has been led to the production of higher antibody titers compared to the control group after a single-dose vaccination [11]. Also, mice immunized subcutaneously with hepatitis B virus surface antigen adjuvanted with chitosan have been reported to give a significantly higher immunogenic response [21].The adjuvant activity of chitosan has been shown for Mycoplasma gallisepticum where chickens intramuscularly vaccinated with the bacterin containing chitosan have exhibited higher antibody response as well as significantly lower tracheal lesions [3].

The mode of action of chitosan in injectable vaccines is only partly explicated. In general, antigen presenting cells take up antigen/adjuvant and transport them to the draining lymph nodes following diffusing away from the injection site. The enhancement of cellular expansion of lymph nodes in the injection site, the retention of antigen in this site and the activation DC are mentioned as the adjuvanticity of chitosan [22]. The ability of chitosan for activation of macrophages and natural killer cells and thus enhancing the immune response is considered as the potential mechanisms of action in its immune stimulation. As a natural carbohydrate polysaccharide adjuvant, chitosan activates DC via a TLR4-dependent mechanism [23]. Chitosan is taken up by macrophages, triggers inflammatory signal transduction and promotes the selective production of type I IFNs using cGAS-STING pathway [24].

Our results showed that chitosan was able to enhance the immune effects of the inactivated IBDV antigen. The antibody 
level induced by two doses of the antigen containing $1 \%$ chitosan was relatively higher than that by the same amount of the antigen containing $0.5 \%$ chitosan. Based on the elevation of neutralizing antibodies in the immunized groups, it is expected chickens which received combinations of the chitosan and the inactivated viral antigens have possessed sufficient protection against IBDV. The serological findings confirmed the adjuvant activity of chitosan to stimulate macrophage populations at the injected site and to increase the efficiency of antigen presentation to the immune cells. It seems that chitosan as a standalone adjuvant recruits cell surface TLR4, modulates the functional activity of the antigen presenting cells, promotes a more efficient uptake of the antigen by DC and activates the expression of cytokines leading to IFN production [25, 26]. As a general trend, chitosan is a potent adjuvant and functionally promotes maturation of $\mathrm{DC}$ as the master regulators of the immune responses by inducing type I IFNs [7]. These cytokines are the critical mediators of chitosan adjuvanticity and promote the maturation and activation of the cells. Maturation begins when DC taking up and presenting antigens to naïve $\mathrm{T}$ cells, which is characterized by enhanced expression of costimulatory molecules [3].

This study emphasized the potential of chitosan as a safe and biodegradable vaccine adjuvant candidate to elicit the humoral immunity against IBDV. Further investigations are needed to evaluate the adjuvant activity of chitosan on decreasing antigen load in the vaccine, uptaking and depot of the antigen, and modulation of the immune responses in the host.

\section{ACKNOWLEDGMENTS}

This study was part of a M.Sc. Thesis in Microbiology, supported by the Razi Vaccine and Serum Research Institute (Karaj, Iran).

\section{CONFLICT OF INTEREST}

The authors declare that there is no conflict of interests regarding the publication of this paper.

\section{REFERENCES}

1. Berg TPVD. Acute infectious bursal disease in poultry: a review. Avian Pathology. 2000;29(3):175-94. doi: 10.1080/03079450050045431.

2. Brandt M, Yao K, Liu M, Heckert RA, Vakharia VN. Molecular determinants of virulence, cell tropism, and pathogenic phenotype of infectious bursal disease virus. Journal of Virology. 2001;75(24):11974-82. doi:10.1128/JVI.75.24.11974-11982.2001

3. Luque D, Saugar I, Rejas MT, Carrascosa JL, Rodríguez JF, Castón JR. Infectious bursal disease virus: ribonucleoprotein complexes of a doublestranded RNA virus. Journal of Molecular Biology. 2009;386(3):891-901. doi: 10.1016/j.jmb.2008.11.029.

4. Müller H, Mundt E, Eterradossi N, Islam MR. Current status of vaccines against infectious bursal disease. Avian Pathology. 2012;41(2):133-9. doi: 10.1080/03079457.2012.661403.

5. Withers DR, Young JR, Davison TF. Infectious bursal disease virusinduced immunosuppression in the chick is associated with the presence of undifferentiated follicles in the recovering bursa. Viral Immunology. 2005;18(1):127-37. doi.org/10.1089/vim.2005.18.

6. Meulemans G. Infectious bursal disease (Gumboro disease). Revue scientifique et technique (International Office of Epizootics). 2000;19(2):527-43.

7. Lima KM, dos Santos SA, Rodrigues Jr JM, Silva CL. Vaccine adjuvant: it makes the difference. Vaccine. 2004;22(19):2374-9. doi: 10.1016/j.vaccine.2003.12.030.
8. Spickler AR, Roth JA. Adjuvants in veterinary vaccines: modes of action and adverse effects. Journal of Veterinary Internal Medicine. 2003;17(3):273-81. doi: 10.1111/j.1939-1676.2003.tb02448.x.

9. Sun B, Yu S, Zhao D, Guo S, Wang X, Zhao K. Polysaccharides as vaccine adjuvants. Vaccine. 2018;36(35):5226-34. doi: 10.1016/j.vaccine.2018.01.029.

10. Illum L, Jabbal-Gill I, Hinchcliffe M, Fisher A, Davis S. Chitosan as a novel nasal delivery system for vaccines. Advanced Drug Delivery Reviews. 2001;51(1-3):81-96. doi: 10.1016/s0169-409x(01)00171-5.

11. Ghendon Y, Markushin S, Vasiliev Y, Akopova I, Koptiaeva I, Krivtsov $\mathrm{G}$ et al. Evaluation of properties of chitosan as an adjuvant for inactivated influenza vaccines administered parenterally. Journal of Medical Virology. 2009;81(3):494-506. doi: 10.1002/jmv.21415.

12. Rauw F, Gardin Y, Palya V, Anbari S, Gonze M, Lemaire S et al. The positive adjuvant effect of chitosan on antigen-specific cell-mediated immunity after chickens vaccination with live Newcastle disease vaccine. Veterinary Immunology and Immunopathology. 2010;134(3-4):249-58. doi: 10.1016/j.vetimm.2009.10.028

13. Volkova MA, Irza AV, Chvala IA, Frolov SF, Drygin VV, Kapczynski DR. Adjuvant effects of chitosan and calcium phosphate particles in an inactivated Newcastle disease vaccine. Avian Diseases. 2014;58(1):46-52. doi: 10.1637/10510-020413-Reg.1.

14. Ebrahimi MM, Shahsavandi S, Masoudi S, Ghodsian N. Isolation, characterization and standardization of new infectious bursal disease virus for development of a live vaccine. Iranain Journal of Virology. 2013;7(4):29-36

15. Miller J, Ulrich R. On the analysis of psychometric functions: The Spearman-Kärber method. Perception \& Psychophysics. 2001;63(8):1399420. doi: $10.3758 / \mathrm{bf} 03194551$.

16. Zhang Z-t, Chen D-h, Chen L. Preparation of two different serials of chitosan. Journal-Dong Hua University-English Edition-. 2002;19(3):36-9. 17. Abdul R, Murgia MV, Rodriguez-Palacios A, Lee C-W, Saif YM. Persistence and tissue distribution of infectious bursal disease virus in experimentally infected SPF and commercial broiler chickens. Avian Diseases. 2013;57(4):759-66. doi: 10.1637/10448-110812-Reg.1 .

18. Petrovsky N, Aguilar JC. Vaccine adjuvants: current state and future trends. Immunology and Cell Biology. 2004;82(5):488-96. doi: 10.1111/j.0818-9641.2004.01272.x.

19. Svirshchevskaya E, Alekseeva L, Reshetov P, Phomicheva N, Parphenyuk S, Ilyina A et al. Mucoadjuvant properties of lipo-and glycoconjugated derivatives of oligochitosans. European Journal of $\begin{array}{lll}\text { Medicinal 2009;44(5):2030-7. } & \end{array}$ doi.org/10.1016/j.ejmech.2008.10.007.

20. Chang H, Li X, Teng Y, Liang Y, Peng B, Fang F et al. Comparison of adjuvant efficacy of chitosan and aluminum hydroxide for intraperitoneally administered inactivated influenza H5N1 vaccine. DNA and Cell Biology. 2010;29(9):563-8. doi: 10.1089/dna.2009.0977.

21. McNeela EA, Jabbal-Gill I, Illum L, Pizza M, Rappuoli R, Podda A et al. Intranasal immunization with genetically detoxified diphtheria toxin induces $\mathrm{T}$ cell responses in humans: enhancement of $\mathrm{Th} 2$ responses and toxin-neutralizing antibodies by formulation with chitosan. Vaccine. 2004;22(8):909-14. doi: 10.1016/j.vaccine.2003.09.012.

22. Zaharoff DA, Rogers CJ, Hance KW, Schlom J, Greiner JW. Chitosan solution enhances both humoral and cell-mediated immune responses to subcutaneous vaccination. Vaccine. 2007;25(11):2085-94. doi: 10.1016/j.vaccine.2006.11.034.

23. Zhang P, Liu W, Peng Y, Han B, Yang Y. Toll like receptor 4 (TLR4) mediates the stimulating activities of chitosan oligosaccharide on macrophages. International Immunopharmacology. 2014;23(1):254-61. doi: 10.1016/j.intimp.2014.09.007.

24. Carroll EC, Jin L, Mori A, Muñoz-Wolf N, Oleszycka E, Moran HB et al. The vaccine adjuvant chitosan promotes cellular immunity via DNA sensor cGAS-STING-dependent induction of type I interferons. Immunity. 2016;44(3):597-608. doi: 10.1016/j.immuni.2016.02.004.

25. Fong D, Hoemann CD. Chitosan immunomodulatory properties: perspectives on the impact of structural properties and dosage. Future Sci OA. 2018; 4(1): FSO225.

26. Mori T, Okumura M, Matsuura M, Ueno K, Tokura S, Okamoto Y et al. Effects of chitin and its derivatives on the proliferation and cytokine production of fibroblasts in vitro. Biomaterials. 1997;18(13):947-51. doi: 10.1016/s0142-9612(97)00017-3. 